\title{
Language Mobile Learning Design: The Tamazight Language
}

\author{
Khadija Achtaich ${ }^{1}$, Habib Benlahmar ${ }^{2,}$ Naceur Achtaich ${ }^{1}$ \\ ${ }^{1}$ Analyze, Modeling and simulation Laboratory, LAMS, Faculty of Sciences Ben M'Sick, Hassan II, \\ Mohemmadia University, Casablanca (MOROCCO) \\ ${ }^{2}$ Information Technologie and Modélisation Laboratory, TIM, Faculty of Sciences Ben M'Sick, Hassan II, \\ Mohemmadia University, Casablanca (MOROCCO)
}

\begin{abstract}
Even though Tamazight language in Morocco has been legitimate for eleven years and official for almost two years, its learning needs a huge effort to successfully make it in this enormous batch of existing unformatted documents. In this article, we present a method for learning the Tamazight language in a mobile environment. The mobile is chosen to free the learner from the constraints of time and space. Indeed, the use of mobile devices allows it to be anywhere and anytime. An immediate question is how to involve the learner in the learning process, which is why we propose to put him in real or fictitious situations that are part of his experience and to which he is used to react. At the end of this article, we also present a prototype of a mobile application implementing our method.
\end{abstract}

Keywords: Learning, Mobile, Situation, Tamazight.

\section{INTRODUCTION}

Information and communication technologies, including mobile ones, break the learning rules and kick it out of the classroom. It's actually learning reborn, with wide horizons, expended perspectives; In a word, with no limits. It can take place in an authentic context and cultural situation, in an informal environment. Neither the time nor the location can command the learner schedule; he can learn anywhere, anytime, all along his life. Also the personal aspect of the mobile device makes the learning more personal and the learner is inside the process. For us, it's evident; we're adopting the situated approach. The learner environment is influencing strategy and drawing the guidelines of our methodology.

In this scoop, the language learning is particularly appreciated and more than ever coveted. A language inter alia should rides on this technology wave, its Tamazight language

With this background of Tamazight situated mobile learning, we catch two targets:

- Assist francophone people (in the first place), from all language/dialect origins, discover some of Tamazight culture and Imazighen civilization.

- Support francophone people (in the first place), from all language/dialect origins, non-Tamazightphone to swap from the phonetic to the transcript using the Tifinagh letter.

This article therefore aims to provide a generic production process for all "usual" situations for the learning. Il will give an entire study of the situation, its components and interactions that manage it.

\section{STAT OF THE ART}

1. Mobile learning: the new discipline.

Mobile learning is still discreetly used in the learning process, shyly known by the random man and rarely used in amateur fields. More than just using the existing technology, mobile learning was a need sated by technological potential of mobile devices such as PDAs, Smartphones, Tablets, etc.

Distance learning concept, also present in the electronic learning, gives its advantages to mobile learning. The mobility concept in newly introduced with this new discipline, none of the classic or traditional learning forms is using neither this axe nor the ubiquity, inherently introduced with mobility.

The evolution of electronic learning to mobile learning is deeply discussed among researchers comity. This transition is associated with a change in terminology which has been widely described by [Laouris Y] in the table below:

Table 1: Terminology in e-learning and m-learning [1]

\begin{tabular}{|ll|}
\hline e-learning & m-learning \\
\hline Computer & Mobile \\
\hline Bandwidth & GPRS, 3G, Bluetooth \\
\hline Multimedia & Objects \\
\hline Interactive & Spontaneous \\
\hline Hyperlinked & Connected \\
\hline Collaborative & Networked \\
\hline Media-rich & Lightweight \\
\hline Distance learning & Situated learning \\
\hline More formal & Informal \\
\hline Simulated situation & Realistic situation \\
\hline Hyper learning & Constructivism \\
\hline
\end{tabular}


Since its introduction, the appellation of mobile is changing all the time. Even today, its definition remains a contestable topic. One of the definitions is the one given by [N.Pinkwart, Al] "Mobile learning is an elearning that uses mobile devices wireless transmission"[2] it tag the mobile learning as a restricted field of the electronic learning. We believe that this definition can be used to describe the thing to novices but certainly not for scientific purposes and research.

MoLeNet1 have a different view and characterizes mobile learning «The exploitation of ubiquitous handheld technologies, together with wireless and mobile phone networks, to facilitate, support, enhance and extend the reach of teaching and learning »[3]. Another definition and probably the most adopted is [O'Malley, Al]'s definition, he defines mobile learning as « Any sort of learning that happens when the learner is not at a fixed, predetermined location, or learning that happens when the learner takes advantage of the learning opportunities offered by mobile technologies »[4]. Two more recent definitions (2010 and 2011) and the ones we will adopt respectively given by [Zhong, Al] and MoLeNet presenting « Mobile Learning is the use of wireless technology-enabled mobile devices for learning at anytime, anyplace and anywhere (...)»[5] «just-intime, just enough and just-for me »[6].

\section{Tamazight}

The Tamazight language is spoken in several African countries, including Morocco, Algeria, Tunisia, Libya, Egypt, Mauritania, Mali and Niger. In Morocco, Tamazight language is legitimate since October 17, 2001 [7] and official since July 2011[8]. The Royal Institute of Tamazight Culture (IRCAM) 2 has the responsibility of promoting the culture and developing the Tamazight language. In collaboration with government authorities and institutions, IRCAM contributes of implementing policies to enable the inception of the Tamazight in the educational system and ensure its raise on both social and cultural levels, in national regional and local standing. Since July 2002, the Tifinagh is officially adopted as Tamazight language alphabet and in June 2004 the Tifinagh is recognized internationally by the international ISO-Unicode.

Tamazight language teaching, however, remains under expectations; it is currently based mainly on a few manuals and documents provided by the IRCAM or the High Commission of Tamazight in Algeria (HCA). These documents are often available physical format (in few specialized libraries) or in electronic format (CDROM, downloaded PDF, or interactive courses for elementary levels).

\section{Architecture}

\section{DESIGN AND FORMALISM.}

In this paper, we propose to describe a scenario of a Tamazight mobile learning process, through a situated learning context. We aim to model the situation, main purpose of our system, which we'll call from now on (the system) all over the paper "Tamazight on Pocket or ToP" for workability reasons.

Therefore, ToP is defined as a situations package, launched, with eventually launch constraints (detailed later), by the learner (consciously or unconsciously). The learner becomes in the middle of the learning process as shown in Fig. 1:

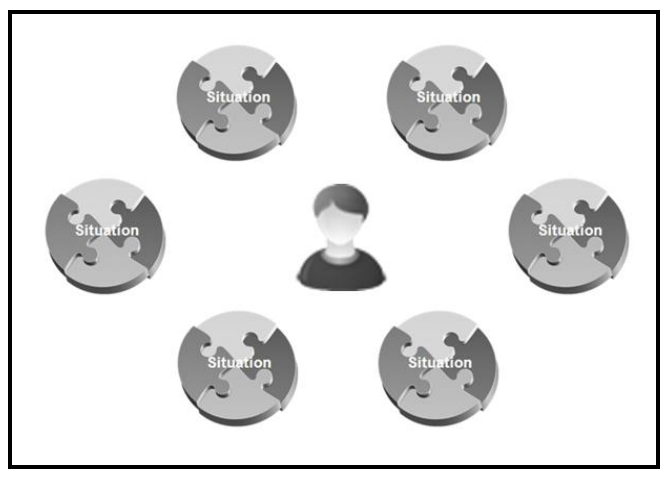

Fig. 1: Learner in the learning process

Situations launch will be handled in another article context; we presume initially that the learner initiates voluntary the chosen situation. The purpose now is to describe the situation, come up with a design for its components and define the collection of interactions managing it. We limit ourselves in this paper to a single typical situation to set example of the generic formalism; at the coffee shop.

1 : Mobile Learning NETwork Un programme britanique pour le support, le dévellopement et la promotion du M-learning.

2 : Academic institute of the Moroccan state in charge of the Tamazight culture promotion and language development. Created by the Dahir (Royal Decree) No. 1-01-299 of October 17, 2011 


\section{Generic design}

Closer, the situation becomes a sequence of phases, more or less important. The order of those phases gives meaning to the situation as perceived in the real world. Fig. 2 shows a basic design of what could view as situation:

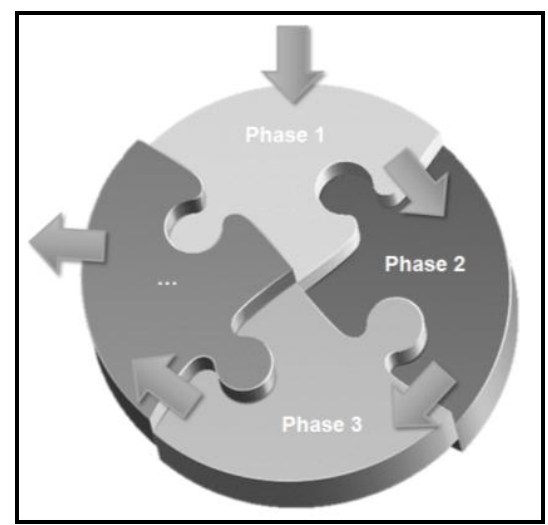

Fig. 2: Phases sequence in a situation

To exploit terminology used for mobile learning, our situation would be a Learning Context (LC). Phases are autonomous entities, able to be (fully or partially) used and reused in several situations. This is exactly how we can define a Learning Object (LO). We're not going to argue or discuss any of the definition given for the LO, we're just announcing the one officially given by the "Institute of Electrical and Electronics Engineers," which defines the learning object as "any entity, digital or non-digital, Which can be used, re-used or referenced falling on technology supported learning". They are supposed to describe the structured learning content and be ready to be consulted by $\mathbf{T o P}$.

A generic design of the LO inspired from the content package design of IMS Global Learning Consortium, Inc. will follow. An LO must have a universal unique identifier. The generic design of LO is mainly built from two parts:

Metadata: Are LO characterizing data, it describes the LO according to both syntactic and semantic aspects. It will give the ToP all needed information to be able to organize LOs in different COs. Metadata can't be exploit as learning content.

Contents: The concrete Learning contents in the LO. It could have a hierarchical structure. The contents are organized in XML elements or links that reference other LOs (or other known structured format as jpg, flv ...)

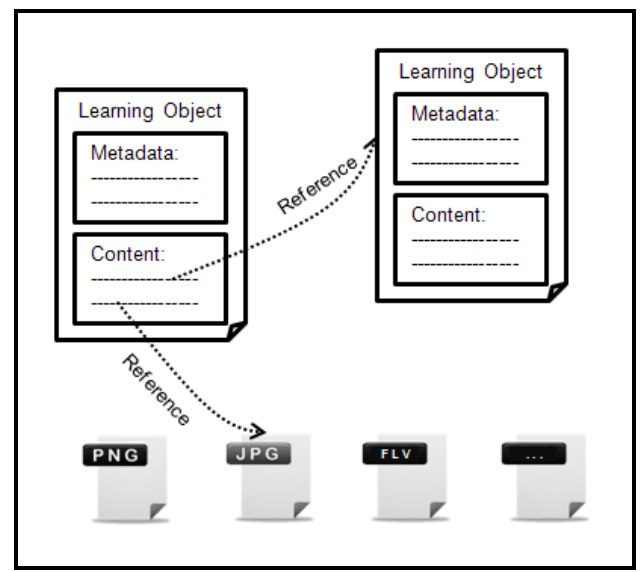

Fig. 3: Generic design of a LO.

The generic design is an abstract design describing all LOs. We decided that two analyzes for LOs from two perspectives were be performed: syntactical analysis and pseudo-semantic analysis. For the syntactical analysis, we investigate the granularity and reference relationships. For the pseudo-semantic analysis, we analyze the educational use of the LO from field use side.

\section{Syntactical analysis}

Syntactical analysis focuses the granularity, the structure and reference relationships of the LO. The goal is to design the LOs, to structure them to attend the aim of an optimum use in an LC. 
We offer three LO splitting levels: Learning Unit (LU), elementary Learning Object (eLO) and complex Learning Object (cLO).

Learning Unit (LU): The lowest split level of a LO, it defines the smallest syntactical granularity in a language: the word. This is the basic element for structuring a LO but can't be employed as a LO because it cannot be used independently for a learning activity. A LU can be referenced in an eLO.

Elementary Learning Object (eLO): The smallest LO that can offer a consistent learning content in a LC. An eLO can be referenced in a cLO. The main purpose for this split level is to improve the LO reuse. The eLO should be as small as possible to fit is unlimited LC. For a learning activity, learning content can be divided into several sub content.

Complex Learning Object (cLO): Can reference one or more eLO and other cLOs. It's the the highest split level. It gives all needed complex structured learning content to use in a LC.

For out typical situation of the coffee shop, syntactical analysis could be as shown in Fig.4.

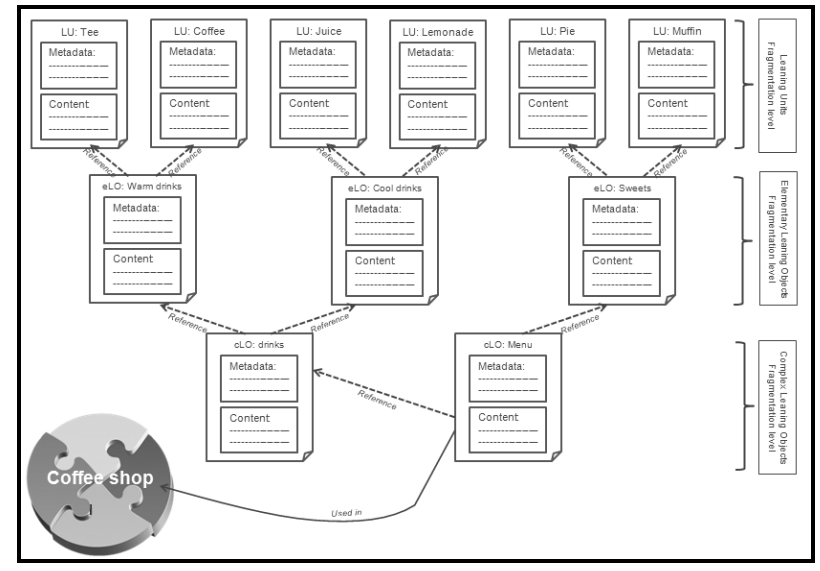

Fig. 4: Syntactical split levels of LO

\section{Pseudo-semantic analysis}

The pseudo-semantic analysis discusses the pedagogical use of the LOs. A LO should be linked a LC according to the learner needs. For example, in the context of bakery, the learner should know or learn the difference between a corn bread, barley bread, etc., in a restaurant however, he only needs to know what the bread is. Through the pseudo-semantic analysis, Los are structured and linked with appropriate metadata that describe their use in a specific LC.

Obviously pseudo-semantic analysis of LOs is not interfering with syntactical analysis. They are both defined from two different points of view on LOs: the granularity level and contextual use of the LO.

The starting point of this analysis on LOs for language learning is the set of lexical items and vocabulary, metaphorically called "Dictionary", edited by specialists or by any Tamazight-phones. For a better design of LOs in an LC, we need to study the characteristics of a real situation, viewed in the real world. Again we took the typical situation of the coffee shop. To fit in the context, we faithfully transcribed the real situation of a young man in a coffee shop. Fig. 5 shows a dialog between the client (the young man) and the waiter. The Dialog goes in dialect Arabic; we translated literally to keep the cultural scope.

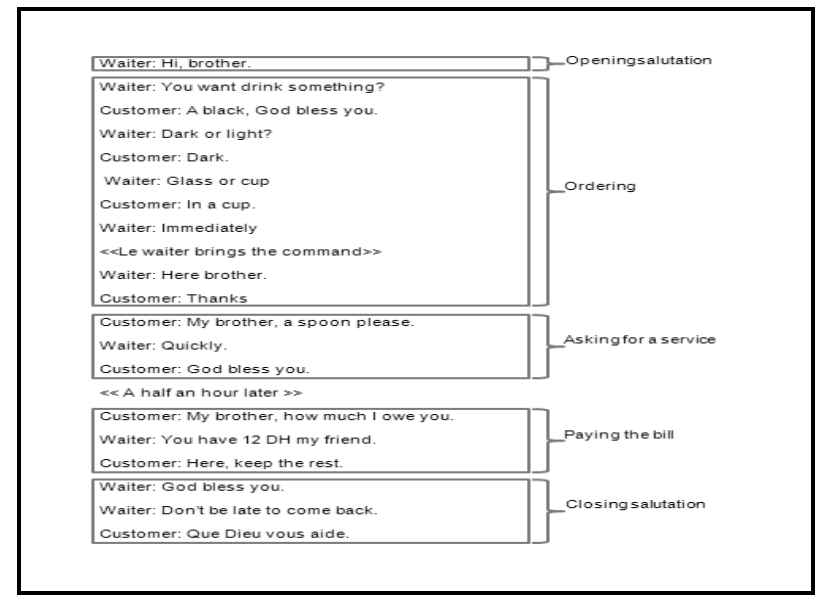

Fig. 5: Dialog in a coffee shop 
With a quick analyze on the dialogue, we can find that it can be divided into several main categories as listed below:

- Opening salutation

- Menu

- Equipment

- Bill

- Closing salutation

That are naturally LOs that we have identified here. Now with dictionaries that we have, we will collect together all items that can be semantically related to the categories above (we will base the collection mainly from the dialogue), we gave a number for each category to ease its referencing. In all the rest of the paper, we'll only use the reference number. All the items used will be used as couple of two atomic parts: item in Tamazight written with Latin alphabet and it's translation in French. Integration of Tifinagh in coming version will follow.

1. Opening salutation $\{$ (Azoul ; Bonjour), (Timnsiwin ; Bonsoir) $\}$

2. Menu \{ (Sou ; Boire), (Lkahwa ; Café), (Lmonada ; Limonade), (Atay; Thé), (Amane ; Eau) $\}$

3. Equipment $\{$ (Taghenjawt ; Cuillère), (Tamskoust ; Chaise), (Lkass ; Tasse) $\}$

4. Bill $\{$ (Lhssab ; Facture), (Fallos ; Pièces), (Idrimnes ; Monnaie) $\}$

5. Closing salutation \{(Tanmirt; Au revoir), (A kdah nzer ; Au plaisir), (Tasbhit tambarkit ; Bonne journée) , (Tadggat tambarkit ; Bonne soirée) $\}$

At this level the plan is to describe an algorithm with two goals: Describe all items that can be included to each LO category and set transitions conditions from an LO category to another. Indeed, the shift from a LO " $\mathrm{X}$ " to a $\mathrm{LO}$ "Y" is not always possible, for example: "1" to "2" is possible, "3" to "2" is possible, "3" to "1" is impossible. The "possibility" of transitions is now discussed; let's see the "conditions" of transitions. If the transition from " $\mathrm{X}$ " to " $\mathrm{Y}$ " is possible, it can occur if and only if one of the items semantically relating to " $\mathrm{Y}$ " is invoked. In other words we will say that we have a set of "steady states" where the learner can stay as long as he didn't invoke a shift "alphabet". The entire "alphabet" used in a LC represents a kind of "language" of this special LC.

We'll indicate by a letter the shift "alphabet" from a random "steady state" to a known "steady state", transition is obviously supposed. From all previous we have the following transitions:
A. Shift alphabet from «X» to «1»
B. Shift alphabet from «X $\mathrm{X}$ to « $2 »$
C. Shift alphabet from «X $\gg$ to « 3 »
D. Shift alphabet from «X» to « 4 »
E. Shift alphabet from «X $\gg$ to « 5 »

We consider all alphabets different: If $\mathrm{I}$ and $\mathrm{J}$ and two different alphabet, then $\mathrm{I} \cap \mathrm{J}=\varnothing$

To summarize, the context of the coffee shop recognizes five "states" to which we add an initial state " 0 " to allow the transition to the " 1 " state, all states will therefore be $\{0,1,2,3,4,5\}$, only the state " 5 " can kick the learner out of context. The language recognized by this context is the language $\mathrm{L}(\mathrm{A}, \mathrm{B}, \mathrm{C}, \mathrm{D}, \mathrm{E})$. With all what has been said, we recognize is a known mathematical formalism; the deterministic finite automaton with " 0 " as a single initial state and " 5 " as a final state.

Fig. 6 shows a general view of the deterministic finite automaton. Every state could be managed by a sub deterministic finite automaton.

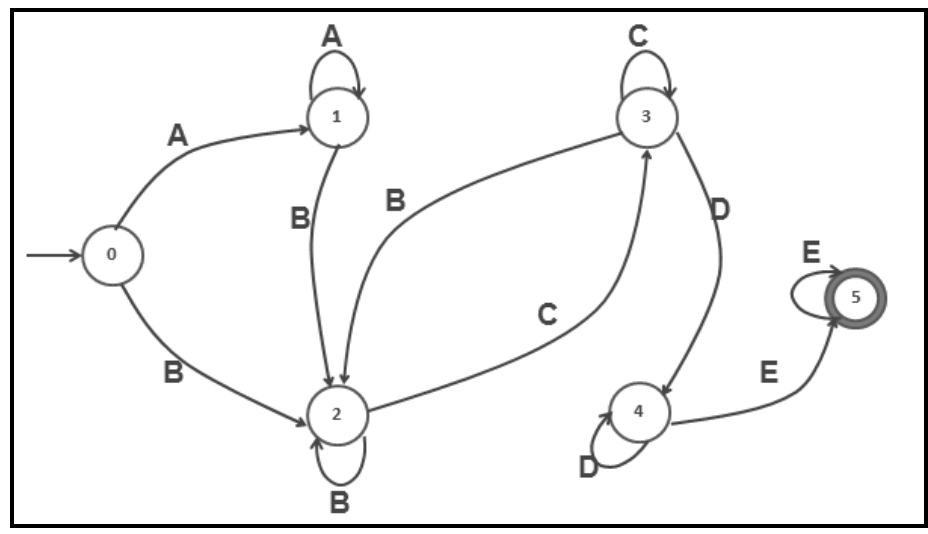

Fig. 6: General deterministic finite automaton 


\section{IV.}

SIMULATION

Fig. 7 shows some screenshots of the prototype we made for our typical situation. First screenshot is the main menu of the prototype; it gives a list of all situations. You can hear the situation name by pressing the microphone icon or enter to the situation when touching its icon. Other screenshots presents the different LOs. At any time, learner have an input field to allow him express himself. Screenwriting is not covered.

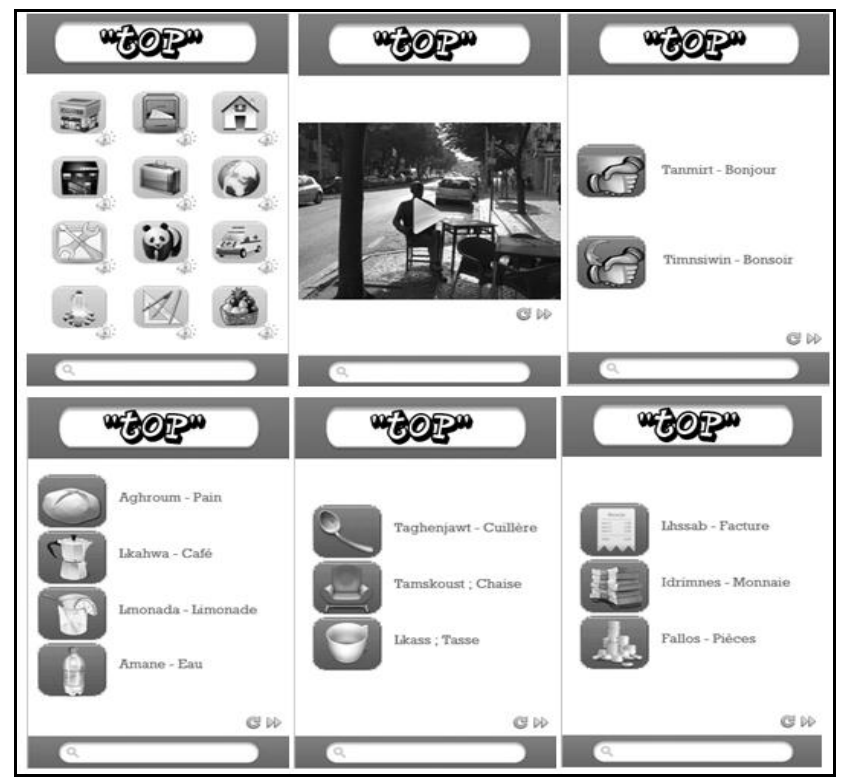

Fig. 7: Screenshots of the ToP application

\section{CONCLUSION AND PERSPECTIVES}

In this paper we focused the definition of a "usual" situation for a learner; we studied its components and the managing interactions, within the context of the Tamazight language learning.

We introduced the puzzling question of producing learning objects within different contexts. We first agreed that a learning object has two main components: the content and metadata. The contents are intended to intercept the organization of learning content; metadata however is used to describe learning objects to grant access to them in different learning contexts.

Meanwhile, learning objects are described from two points of view: syntactical and pseudo-semantic. The syntactical description points the structure and the granularity of the learning object. We have three hierarchical levels of learning objects: the learning unit, the elementary learning object and the complex learning object. The pseudo-semantic description focuses instead the learning objects content and their pedagogical contexts of use, it discuss both homogeneity of the learning object, its implication in the learning context, as well as intra learning objects transition management.

A typical situation of coffee-shop has been represented using both syntactical and pseudo-semantic description. However it was all manually evaluated. In fact, metadata are not used, we simply announce them as one of the main component of the learning object. A study on metadata, their type and their involvement level will be the topic of a future paper.

Finally, we proposed a prototype to simulate the result of the work cited above. At this level we came with all items in Tamazight (written in Latin letters) and translated into French. The introduction of the Tifinagh letter is on development level.

\section{REFERENCES}

[1] Laouris, Y. (2005). We need an educationally relevant definition of mobile learning

[2] Pinkwart, N, Hoppe, H.U, Milras, M and Perez, J. (2003). Educational scenarios for the cooperative use of Personal Digital Assistants. Journal of Computer Assisted Learning, pp. 383-391

[3] MoLeNET. (2009). Seen on www.molenet.org.uk, May 2nd, 2012 (2009)

[4] O’Malley, UoN, C, Vavoula, G, UoB, J.P. Glew, UoB, J. Taylor, OU, M. Sharples, UoB, P. Lefrere, (Juin 2003)

[5] Zhong, L. Xue, Q., Liu, H., Yuan, J. (2010). Research on 3G Mobile Learning Based on Cloud Service, IEEE, International Conference on E-Product E-Service and E-Entertainment (ICEEE) , pp. 1-4.

[6] MoLeNET. (2011). Seen on www.molenet.org.uk, May 2nd, 2012 (2011)

[7] Reference to the king's speech in Ajdir At October 17, 2001

[8] Constitution of Morocco. (July 2011). Paragraph 1, Article 5. 